Box 1: Single-molecule force measurement $\quad 874$

Box 2: High-throughput force measurement $\quad 875$

\title{
Pulling on single molecules
}

\author{
Natalie de Souza \\ Single-molecule methods to apply and measure force and displacement are expanding mechanobiology.
}

Force is central to biology. Motor proteins apply force to move cargo within the cell. Adhesion molecules at the cell surface register tension in the surrounding matrix. Specialized tissues such as muscle sense mechanical stimulus and do mechanical work. Tiny forces within and between molecules control their shape and keep molecules bound to each other. Over the past decades, there has been substantial development of methods to apply miniscule forces to single molecules and to measure the molecule's response. These methods are leading to a finer-grained picture of the mechanics of biological function.

\section{Molecular mechanics in blood}

Blood clotting is not the first process that comes to mind when considering what one might study by pulling on single molecules. But mechanics matter at many scales: applying force to a molecule can be informative not only about its own molecular activity but also about cell or tissue function. In a recent elegant example, Timothy Springer and colleagues at Harvard Medical School have used singlemolecule force measurements to show that a molecular bond between clotting factors has unique properties that may be physiologically relevant at the macro scale.

Von Willebrand factor (VWF) is a critical clotting protein in the blood; it binds to platelets, and is important for platelet clumping and for their interaction with the wall of a blood vessel. Springer and colleagues studied the in vitro interaction of a domain of VWF with glycoprotein $1 b \alpha(G P 1 b \alpha)$, its receptor on the platelet surface. They tethered the end of each molecule to a separate immobilized bead and used the resulting 'tweezers' to apply tiny forces to pull the proteins apart.

The two proteins, they discovered, inter- act in an unusual way. The bond between them has two states: one predominates at lower force (below 10 piconewtons $(\mathrm{pN})$ or so), but at higher force, a second, stronger state appears. Springer and colleagues define this as a 'flex bond'. (Other unusual bonds like the so-called catch bonds have been observed-also by single-molecule experiments - to operate between some adhesion molecules and their ligands.) The flex bond between VWF and GP1b $\alpha$ could be one way to ensure that the clotting factors can interact over a range of forces, which may help to keep clotting robust.

\section{Using force on molecular bonds}

It was not always obvious that applying force to single molecules could be used to study molecular bonds. "At the beginning, force spectroscopy was rather explorative," remarks Daniel Müller at the ETH Zürich, who makes force measurements on membrane proteins at the cell surface. "The understanding that molecular interactions can be measured in terms of forces, this had to develop."

The techniques used to apply force to single molecules - the main ones are optical tweezers, magnetic tweezers and the atomic force microscope (Box 1)-have migrated to biology from the physical sciences over the past decades. By the late 1980s, Arthur Ashkin and colleagues at Bell Labs had demonstrated the use of optical tweezers to trap and manipulate bacteria, viruses and eukaryotic cells.

At the molecular level, optical traps were used in the early 1990s by Steven Block and colleagues, then at Harvard University, to measure the force exerted by motor proteins as they moved on microtubules. The extension of DNA under force, in turn, was being measured by Carlos
Bustamante and colleagues, then at the University of Oregon, Eugene. In 1994, two independent groups, that of Herman Gaub, then at the Technische Universität, Munich, and that of Gil Lee at the Naval Research Laboratories, Washington, DC, measured the strong intermolecular interaction between avidin and biotin using an atomic force microscopy (AFM)based approach. "From there I think we started to think differently," says Müller. "Here was a clear biochemical interaction between a receptor and a ligand, and force was used to characterize this interaction."
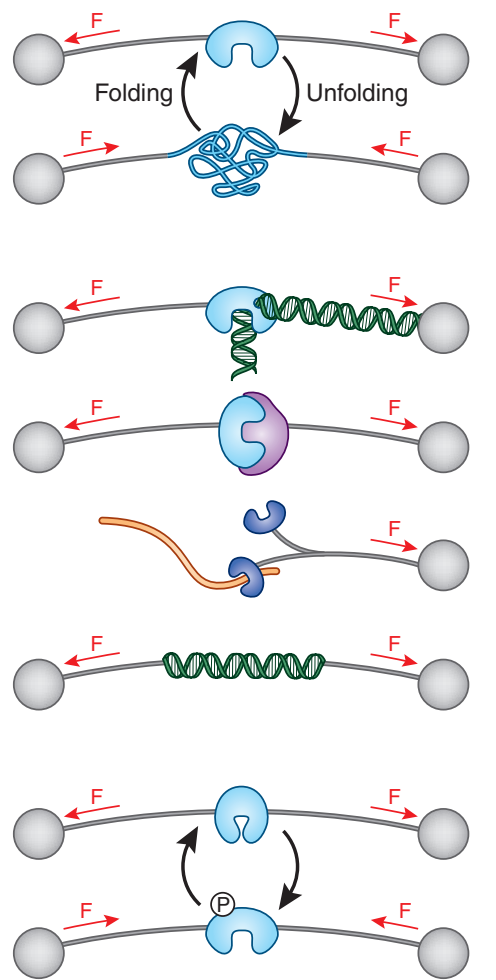

Single-molecule force measurements are used to study protein or DNA structure, receptor-ligand or DNA-protein interactions, enzyme activity, or molecular motor function. Modified from ref. 1. 


\section{The importance of theory}

As the instruments used in force spectroscopy improve and the measurements become more precise, advances in the field are increasingly being driven by theoretical developments, says Keir Neuman at the US National Institutes of Health, who uses magnetic tweezers to study the structure and topology of DNA. One recent instance is the analytical framework developed by Olga Dudko, now at the University of California, San Diego, and her colleagues, for interpreting single-molecule force experiments. The Dudko-Hummer-Szabo

\section{BOX 1 SINGLE-MOLECULE FORCE MEASUREMENT}

The three main methods for singlemolecule force measurementsoptical tweezers, magnetic tweezers and atomic force microscopy (AFM)based molecular force probes-all involve variations on the following theme: the molecule of interest is tethered to a surface at one end and attached at its other end to a probe, through which force is applied. The probe is typically a polymeric or magnetic bead (optical or magnetic tweezers), or a cantilever (AFM). Force is applied via optical or magnetic interactions or by mechanically retracting the AFM probe. Molecular extension, bond rupture or activity-coupled movement is detected by tracking displacement of the probe relative to the tethering surface. It is critical that the position of the probe is precisely determined and that the system's response to force is calibrated. The experiments are extremely sensitive to fluctuations in the environment.

In an optical trap, the bead experiences a restoring force toward the focal point of a highly focused laser beam. 0ptical traps have a force range of $0.1-100$ piconewtons $(\mathrm{pN})$ and can measure displacements over 0.1-10 5 nanometers ( $\mathrm{nm}$ ).

In magnetic tweezers, the bead is pulled by a magnetic field gradient generated with permanent magnets or electromagnets. Magnetic tweezers can be rotated and are well suited to study phenomena, such as DNA topology, where twist has a role. They have a force range of $10^{-3}-10^{4} \mathrm{pN}$ and can measure displacements over $1-10^{5} \mathrm{~nm}$.

In an AFM-based setup, the cantilever tip is brought physically in contact with the tethering surface and is then retracted. The cantilever bends until the tethered molecule extends or bonds break. AFM-based force probes have a force range of $10-10^{4} \mathrm{pN}$ and a distance range of $0.5-10^{4} \mathrm{~nm}$.

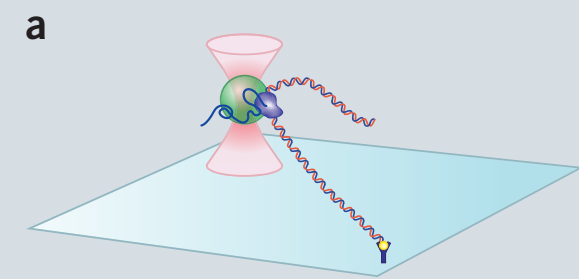

b

C

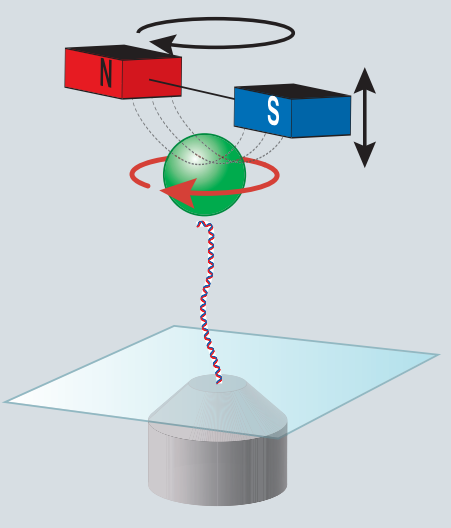

Detection laser

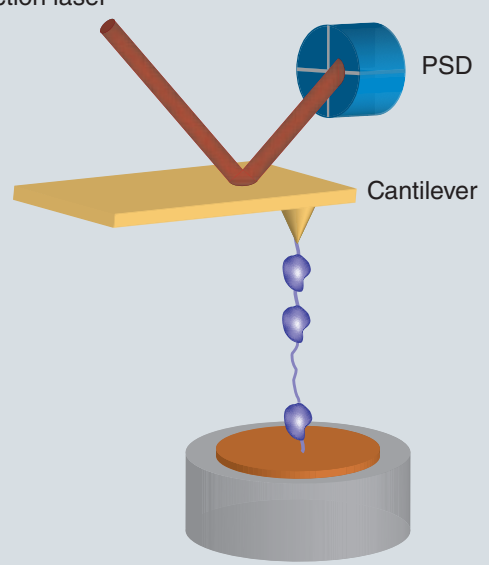

Piezoelectric scanner

Methods for single-molecule force measurements. (a) An optical trap. (b) A magnetic trap. (c) An AFM-based force probe. F, force. Modified from ref. 3. 
framework goes beyond the pioneering Bell-Evans model, developed by Evan Evans at Boston University, and overcomes some of its limitations, Dudko says.

The results of single-molecule pulling experiments can be fit to Dudko's theory to map the key features of a molecular energy landscape. One can obtain the rates of a molecular transition-for instance, of receptor-ligand association and disassociation, of nucleic acid unzipping and rezipping or of protein unfolding and refolding-as well as the height and the location of the rate-limiting barrier to the transition. Steven Block, at Stanford University, in recent work on the adenine riboswitch from Bacillus subtilis, and Taekjip $\mathrm{Ha}$, at the University of Illinois UrbanaChampaign, in a study of protein-DNA binding, have both made use of this theory to interpret their data, as did Springer in his work on clotting factors.

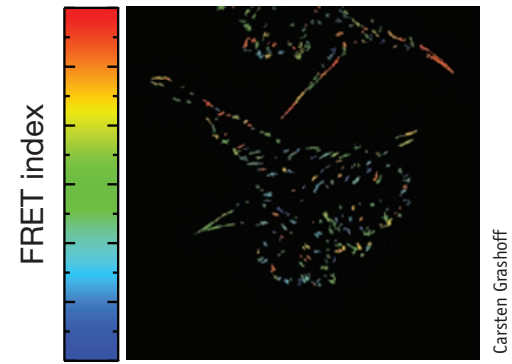

A fluorescent tension sensor in vinculin in an endothelial cell. Reprinted from ref.2.

Dudko is quick to point out that every model has limitations and that the processes biophysicists are studying are complex. "The more we learn about these processes the more I realize how little in fact we know," she says. For instance, her theory applies to transitions over a single rate-limiting energy barrier. Yet energy landscapes of many real biological

\section{BOX 2 HIGH-THROUGHPUT FORCE MEASUREMENT}

It is perhaps not widely appreciated outside of biophysics laboratories that single-molecule force spectroscopy requires the collection of vast amounts of data. "Not only do you need many measurements to get the statistics to be able to interpret your data," says Rachel Owen, Senior Application Scientist at JPK Instruments, a leading manufacturer

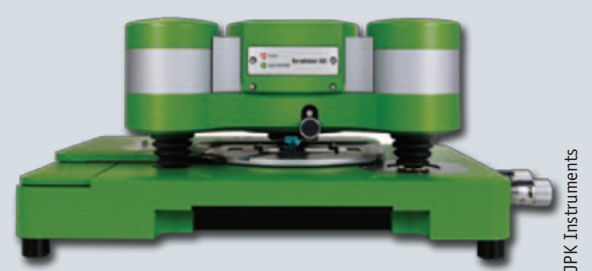

The ForceRobot 300 from JPK Instruments. of force-measurement devices, "but if you're looking at a single molecule, only about $10 \%$ of the measurements should even give you a positive signal."

What is more, making these measurements with an AFM-based force probe can be very monotonous: parameters that need adjustment are rarely interesting ones that require creative decisions. "You plan an experiment to measure under various conditions, and you just want the instrument to take care of it," Owen says. Small wonder then that there has been a push both in industry and academia to automate the process.

JPK released its ForceRobot 300 in 2009. The instrument is an automated AFMbased force probe that can measure and analyze tens of thousands of single-molecule force-extension curves in a few hours, unsupervised and around the clock. "It even sends you an SMS once it's done," says Daniel Müller, who has collaborated with JPK in the development of the robot and has one in his lab. "The only problem is now my graduate students have to find something else to do," he jokes.

Wesley Wong at Harvard University has also tackled this problem, using a different strategy. Wong and colleagues built a centrifugal force microscope in which an entire tiny microscope is spun, thus applying a controlled and calculable centrifugal force to beads tethered to a coverslip via the molecule of interest. When the device is spun, the beads are displaced off the coverslip to a position equivalent to the length of the molecular tether. Bond rupture moves the bead out of focus, and this can be detected in an automated fashion. Wong has used the centrifugal force microscope to make thousands of parallel single-molecule force measurements on digoxigenin bound to its antibody. 
systems contain multiple kinetic barriers. Prompted by experimentalists, Dudko and her group are working toward a theory that could be used to interpret experiments on more complex systems.

\section{Mechano-enzymatics}

The most obvious subjects for singlemolecule mechanical measurements are mechano-enzymes that themselves generate force or displacement. These molecular machines-motor proteins of the kinesin, myosin and dynein families or enzymes such as DNA and RNA polymerases that move along nucleic acids in motor-like fashion-use the energy of ATP hydrolysis (or of ionic gradients, in other cases) to transport, remodel or denature other macromolecules or biological structures.

For motors, force-spectroscopic studies typically are used to determine the so-called stall force, the force that must be applied to a motor to prevent it from moving cargo, but they also reveal information about the chemical-mechanical coupling of these enzymes; kinesin, for instance, was shown by Block and colleagues to take an 8-nanometer step with the consumption of a single ATP. Not all molecular machines are so thrifty: the AAA+ protease ClpX, which mechanically unfolds proteins before it cleaves them, can burn hundreds of ATP molecules if faced with a difficult substrate to unfold, as observed by Robert Sauer, Tania Baker, Matthew Lang and colleagues at the Massachusetts Institute of Technology.

But macromolecules do not only generate force. Some also sense it, and in some cases go on to transduce it into enzymatic activity that controls downstream processes. Single-molecule force measurements are increasingly used to study such force-sensing enzymes, too. In studies of the muscle protein titin, for example, Elias Puchner, now at Stanford University, with Mathias Gautel, at King's College, London, and their colleagues, have combined AFM-based force measurements with molecular dynamics simulations to study titin kinase activation by mechanical force.

And the reach of single-molecule force measurements in the study of enzymes goes even beyond those that explicitly sense or generate force. Disulfide bond reduction catalyzed by thioredoxin, for instance, is affected when the substrate is subjected to force, as observed by Julio Fernandez and colleagues at Columbia
University. As Block puts it, "Force and motion and tiny displacements are a really important part of every enzyme. Of course the notion of allostery is not new [...] but only recently has it been possible to study it from [...] the biophysical perspective."

\section{Mixing force and fluorescence}

Powerful though they are (Box 2), force measurements do have drawbacks. For one, they typically report only on molecular movements that occur along the pulling axis. It also remains difficult to measure small molecular displacements at low force; the tether that connects the molecule of interest to the force probe is typically too flexible to be reliable in such cases. To overcome some of these problems, a second measurement mode, such as fluorescence, can be incorporated.

Broadly speaking, this can be done in two ways. First, the molecule to which force is applied can be simultaneously monitored with fluorescence, as was accomplished by $\mathrm{Ha}$ and colleagues on studies of the Holliday junction (a four-armed DNA structure that forms during recombination) or by Jan Liphardt and colleagues at the University of California, Berkeley, on studies of short single-stranded DNA loops. Both studies used fluorescence resonance energy transfer (FRET) between incorporated fluorophores to monitor molecular structure under applied force.

Alternatively, fluorescence can be used as a direct readout of the force that a biological molecule experiences. Khalid Salaita and colleagues at Emory University recently used polymerbased fluorescence sensors to monitor the mechanical tension on specific cell-surface proteins. In separate work, Martin Schwartz and colleagues at the University of Virginia, Charlottesville, placed a domain from flagelliform-a protein in spider silk that forms a tiny force-sensitive spring-between two fluorescent proteins that undergo FRET. They spliced the construct into vinculin, a protein of the focal adhesion complex, expressed it in cells and monitored FRET. Calibration of the sensor in vitro, in collaboration with $\mathrm{Ha}$ and his team, yielded force-fluorescence curves that could be used to read out the force experienced by cellular vinculin. 
Ha points out that these calibrations make some assumptions. "We are using organic dyes to calibrate and in the cell we are using fluorescent proteins. I would say the accuracy of the calibration is within about 30\%," he says. Also, the original sensor was only sensitive up to about $5 \mathrm{pN}$, whereas relevant biological forces are likely to be as high as $10 \mathrm{pN}$ or even $20 \mathrm{pN}$. Ha and his colleagues have designed shorter sensors that report on force up to $10 \mathrm{pN}$.

\section{Force measurements in vivo}

With the exception of AFM-based measurements on cell-surface molecules, most single-molecule force measurements are conducted in vitro. How do these measurements correlate with molecular behavior within the cell? As Neuman puts it, "We now have very good control in vitro over DNA tension and twist, so we can understand its structural changes very well. But what are the forces acting on DNA during replication, during cell division? What's the tension of that DNA? There simply haven't been very good measurements of these forces."

Both optical and magnetic tweezers have been applied inside of cells, but these experiments remain challenging. In most cases beads must be introduced into the cell and the experimenter must contend with unwanted perturbations of cellular or molecular function, for instance, local heating by optical tweezers. Notably, the control over the environment afforded by in vitro experiments is lost inside the cell: in vivo measurements are much more prone to nonspecific interactions and require more careful design and interpretation. Finally, calibration in vivo is far from trivial.

Block comments that in vivo force measurements are "an inevitable direction for the field." Nevertheless, he cautions that the practical difficulties in carrying out such experiments should not be underestimated. Taekjip Ha in turn argues that in vitro work, with its greater control over experimental conditions and measurement modes, may paradoxically be the more direct route to making more physiological measurements. "In my view we need what I call extreme in vitro," Ha says. "If you just have one molecule and you're pulling on it, it doesn't tell you the whole story. You want to look at complexes. For that you want to measure multiple parameters at the same time, and the combination of force and multicolor fluorescence [at very high resolution] will be critical."

Single-molecule force measurements are only a few decades old and are as yet performed in a relatively small number of laboratories. Yet their reach into multiple biological arenas-structural biology, enzymatics, cell biology, even physiology-is already palpable. They reveal, it could be argued, that the millenia-old notion of the body as a complex machine applies perhaps most neatly to its constituent molecules.

1. Dufrêne, Y. et al. Nat. Methods 8, 123-127 (2011).

2. Grashoff, C. et al. Nature 466, 263-266 (2010).

3. Neuman, K. \& Nagy, A. Nat. Methods 5, 491505 (2008).

Natalie de Souza is a senior editor at Nature Methods (n.desouza@us.nature.com). 